\title{
Mobile-based Monitoring of Parkinson's Disease
}

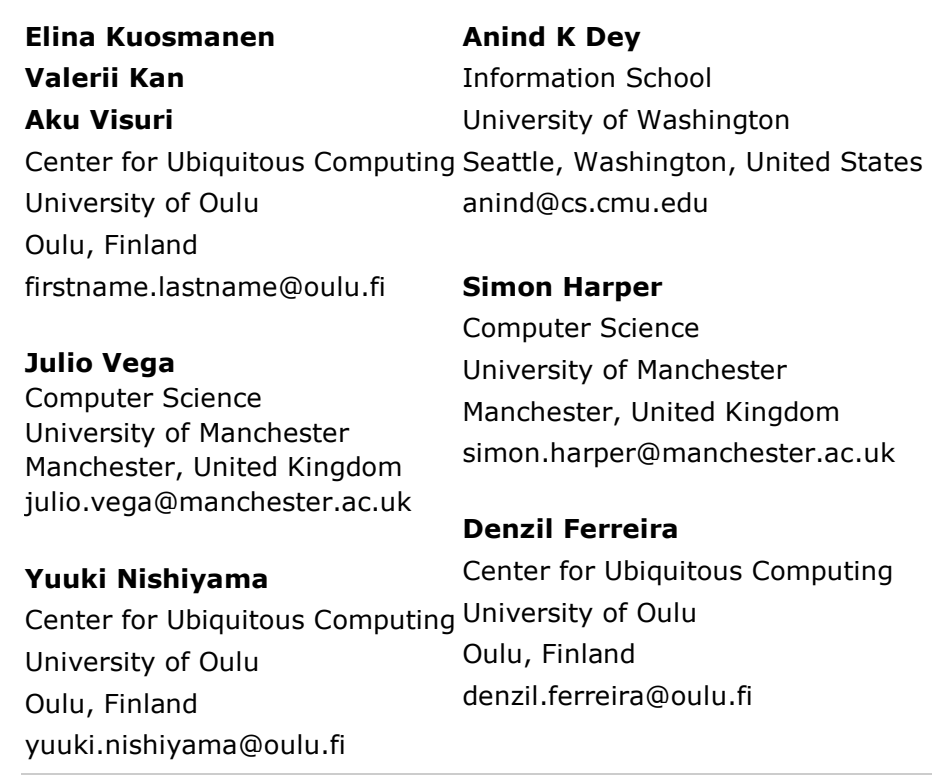

Permission to make digital or hard copies of all or part of this work for personal or classroom use is granted without fee provided that copies are not made or distributed for profit or commercial advantage and that copies bear this notice and the full citation on the first page. Copyrights for components of this work owned by others than the author(s) must be honored. Abstracting with credit is permitted. To copy otherwise, or republish, to post on servers or to redistribute to lists, requires prior specific permission and/or a fee. Request permissions from

Permissions@acm.org.
MUM '18, November 25-28, 2018, Cairo, Egypt @ 2018 Copyright is held by the owner/author(s). Publication rights licensed to ACM. ACM ISBN 978 $1-4503-6594-9 / 18 / 11 \ldots \$ 15.00$

https://doi.org/10.1145/3282894.3289737

\begin{abstract}
Parkinson's disease (PD) is the second most common neurodegenerative disorder, impacting an estimated seven to ten million people worldwide. It is commonly accepted that improving medication adherence alleviates symptoms and maintains motor capabilities. Not following the medication regimen (e.g., skipping or over-medicating) may worsen side-effects, which mislead clinicians and patients. We developed and evaluated a mobile application, STOP, for screening the PD symptoms and medication intake. It contains a game for tracking the PD symptoms, and a medication journal for recording medical intake and adherence. We conducted a 1-month long real-world deployment with 13 PD patients from two countries. We found that the application medication adherence tracking provides non-bias information, and users are receptive to share such data with their care and medical personnel.
\end{abstract}

\section{Author Keywords}

Parkinson's disease; smartphone; gamification;

logging; empirical evaluation

\section{CCS Concepts}

- Information systems Mobile information processing systems 


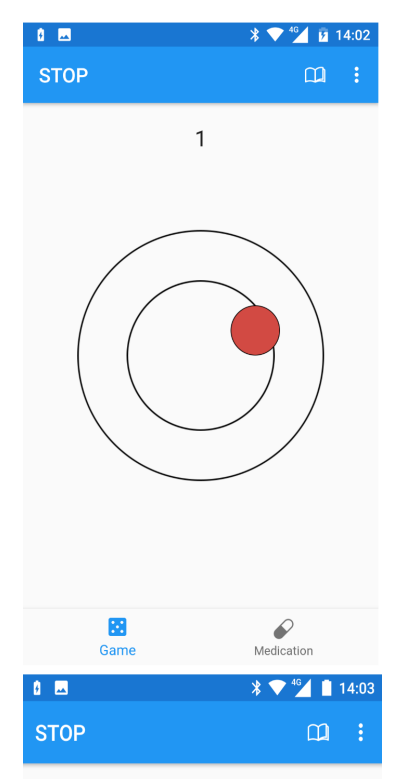

Done! Play again?

Score: 67.9 , last score: 84.6

Now record your medications

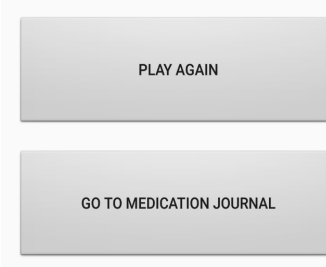

因

Figure 1: Ball game interface

\section{Introduction}

Parkinson's disease (PD) is a progressive neurological disorder with both motor and non-motor symptoms having a major impact on patients' quality of life. Effective medication for alleviating the symptoms exists [15], nevertheless, prescribing patients the right doses of medication is challenging. The diversity of drugs and the doses patients need increase along the progress of the disease. PD patients visit their doctor typically twice a year, even though their symptoms can fluctuate within hours or days. Understanding these fluctuations are crucial for patients and clinicians to track and monitor the effectiveness of the current medication. Keeping a medication diary is burdensome, and to recall details on medication intake after months is challenging. Tracking PD with a mobile app can improve medication adherence, and the collected data can improve the quality of clinical consultation [10].

Therefore, our main objective was to create a mobile tool for PD patients for assisted medication logging to improve adherence, and also to understand the lasting effects of the current medication regimen. We developed and evaluated a mobile application, STOP $[8,9]$. For monitoring motor PD symptoms, STOP features a ball-balancing game utilizing smartphone's motion sensors (e.g., accelerometer, gyroscope) and an electronic journal for assisted recording of medication intake, to act as a tracker for medication adherence. Our hypothesis is that STOP would facilitate in some degree the management of PD.

\section{Related Work}

Not exclusive to only PD, medication non-adherence is a common problem that lowers the effectiveness of a medication-based treatment, and triggers (unnecessary) medication regimen changes [7]. It has been shown that symptoms can be mitigated, and motoric capabilities improved by effective medical adherence [5]. Smartphones contain a wide variety of inbuilt sensors useful for PD observation. The severity of the patients' tremor has been evaluated using an iPhone attached to a glove [11] and to classifying essential tremor and PD tremor $[3,16]$. The mPower study collected both PD and non-PD participants' data via health surveys and physical activities for 14 days every three months $[1,2]$. This dataset contains selfreported symptoms, medication logs, and miscellaneous tasks performance (collected using the phones' sensors data) [4]. Several studies used this dataset, i.e., Neto et al. [13] analyzed personal medication response on daily routines from the sensor data collected.

\section{STOP: Mobile-based Tracking of Parkinson's}

STOP is a smartphone-based application for PD patients for tracking symptoms and medication $[8,9]$, available for Android and iOS. STOP's core functionalities are: a ball game that quantifies patient's motor symptoms, a medication journal where users log their medication intake time, and a daily survey for reporting the overall severity of PD symptoms.

Ball game: The goal of the ball game is to balance the smartphone horizontally with the screen facing up on the palm of the hand for a chosen amount of time ( 10 seconds in our evaluation, empirically determined in [8] with healthy participants), keeping the ball at the center of the screen, inside the inner circle (Fig. 1 upper). The success is evaluated by score between $0-$ 100. If the ball is kept within the inner circle perfectly centered, the score is 100 . If the motor symptoms 

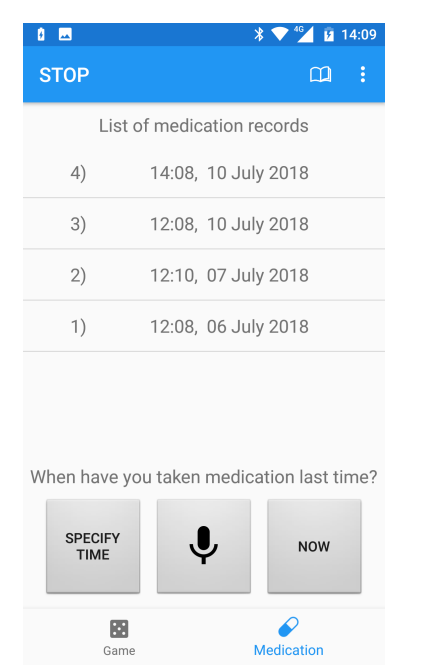

Figure 2: Medication journal

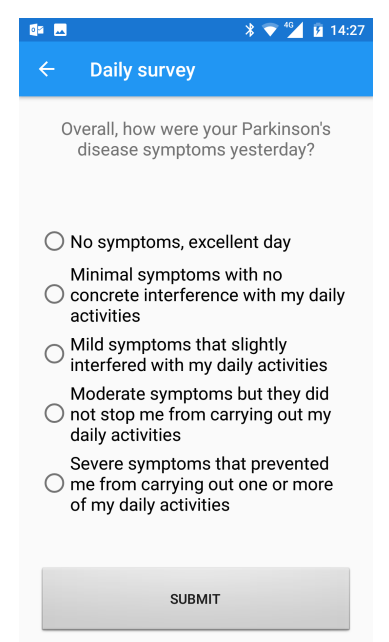

Figure 3: Daily symptom survey prevent user to keep the ball motionless, the score decreases. To keep the game engaging, we randomized the ball sensitivity four times per day between 1 (slow) and 5 (fast) in the Android application. We kept the ball sensitivity the same on iOS.

Medication Journal: The time of medication intake is recorded to medication journal using manual input ("Now" and "Specify time" buttons) or via the voice input, powered by the natural language processor [12] (Fig. 2). Every morning between 10.00 and 11.00 , the user is asked to evaluate the level of PD symptoms on previous day (Fig. 3). Notifications are used as reminders to regularly play the game and record medication intake times, and to deliver the daily symptoms' survey.

\section{Experiment}

We conducted an in-the-wild trial for the application for obtaining insight into the implications of our design and collecting user experiences. We recruited participants from two countries, Finland and UK, for minimizing the population selection bias, leveraging local Parkinson associations for advertising the study. The study required the use of the STOP for a 1-month period and three interviews (before installing STOP, half-way, and at the end). For eligibility, the participants had PD clinically diagnosed, own a smartphone (e.g., Android $4.4+$, or iOS $10.0+$ ), and have internet connectivity on the smartphone or over $\mathrm{Wi}-\mathrm{Fi}$ at home. A reward of a store gift card (100 Euros in Finland; 50 Pounds in UK) was given at the end for successful participants. The amount was different due to budget availability differences.
In three months, we succeeded in finding 14 eligible participants, eight from Finland and six from UK, nine females and five males. One left the study after the initial interview. Five users had iOS device, eight used Android. The age range of the participants was 52-73, with PD diagnosed 2-17 years ago. The amount of different PD medicine intake varied from 1 to 5 , and daily medication taking 1 to 7 times per day. Three participants had a Deep Brain Stimulator (DBS). Using the UPDRS scale II score (Unified Parkinson's Disease Rating Scale, part II)[6], participants self-evaluated their symptoms, with scores ranging from 3 to 31 .

The study's consent form was within STOP and it was approved by respective IRB Ethics Committees. The participants in UK signed a paper version. Our team is certified on HIPAA, GDPR and ethical research guidelines. The collected data was sent regularly and securely to our hosted server over Wi-Fi or cellular data connection. Each participant was identified via a randomly generated 128-bits Universal Unique ID (UUID). Quitting the study was allowed by the application at any time, deleting the collected data from the device and from the server. For practicality and minimize the burden of mobility, in Finland the application was delivered via Google's Play Store for Android devices and Apple's App Store for iOS devices, and the study guide including screen captures and an overview of the functionalities of the app was sent by e-mail. In UK, we had an initial face-to-face interview where we installed the application and walked through the study guide.

\section{Collected Data}

The following data were collected by the STOP application: 
- Patient characteristics: at first launch, we record the participant's age, how long-ago PD was diagnosed, which PD medications are taken, and a self-evaluation of PD symptoms using UPDRS part II scale.

- Game data: Scores, and mobile device sensor data during the game sessions using four smartphone's sensors - accelerometer, linear accelerometer, gyroscope and rotation.

- Medication time data: The medication intake time is manually recorded in the journal.

- Notification data: When the notifications are triggered and when they are opened.

- Feedback: Users' feedback, sent via the app.

In the initial interview, conducted before installing STOP, we focused on understanding the daily life struggles due to PD and what challenges it brings to mobile device usage. The half-way interview focused on STOP usage feedback. In the debriefing interview we inquired on perceived benefits of using the STOP, and its impact on medication adherence.

In the one-month deployment, the 13 participants recorded 1116 medication logs and 1797 game sessions.

\section{Results and Discussion}

Based on our interviews, we got an understanding how PD challenges the participants' daily lives. Medical and dietary regimen dominate the daily life, and the fluctuating symptoms cause difficulties:

"I manage somewhat ok from all my tasks. I live alone, so I do everything by myself. Lately I have become slower and it is slower to start moving, I get winded more easily, there is pain and hair loss. Few years ago, I started to notice rigidity in some movements, I stopped and noted that I need to consciously send a message from my brain: 'move the leg' or 'lift the paper' or when skiing 'hit the stick to the ground'." (P1)

Some of the participants have learned to live with PD, while others experience the symptoms as restrictive. Surprisingly, the younger patients, and those with shorter PD history seemed to experience the symptoms as more disturbing in their daily lives, affecting their work or family life. The difficulties in the mobile phone use were more cohesive, mainly related to text input. Participants used voice input instead of typing or used a stylus instead of finger.

Adherence: We measured the time between the schedule and actual reported medication intake and used that as a measure for medication adherence. However, so called "boosters", the extra medication that can be taken if needed, they are not included to the schedule. In addition, some medication does not have a strict timed schedule (e.g., must be taken exactly at $8 \mathrm{AM})$, as it can be taken after waking up (depending on when the patient wakes up), or when going to bed. Sometimes a participant decides to take the medicine earlier or later, if he has demanding activities, such as going to physiotherapy. Nunes and Fitzpatrick [14] discuss in their paper whether this is considered as non-adherence, due to patients being aware of the consequences of deviating from schedule, but wanting be at their best performance in the activity.

From the data (Fig. 4) we observed that the adherence of two participants (P4 and P9) was slightly improved during the study. On the other hand, there were also 
two participants (P2 and P10) whose adherence decreased. However, most participants maintained their adherence.

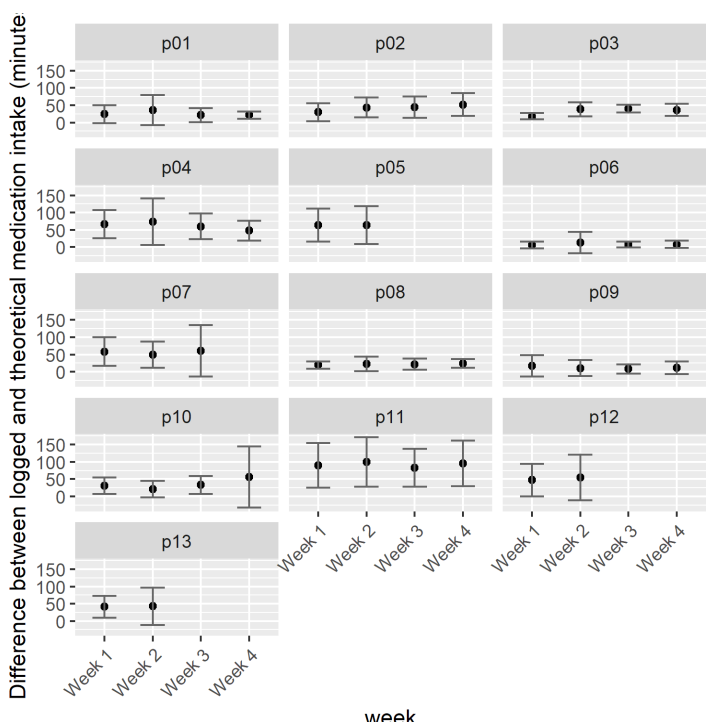

Figure 4: Weekly time adherence of the participants in minutes

Only two participants mentioned they felt their medication adherence had improved during the study, however, surprisingly these were not the ones who had improved the adherence based on the collected data. One participant (P11) commented in the half-way interview that recording the medication time made him notice how irregularly he takes his medication. He thought that's partially because, at this moment, his PD symptoms are not that severe.

Gamification: the participants were prompted to play the game at least four times per day during the study period. The assumption was, that from the motor symptoms, tremor has the biggest effect on the score. From UPDRS survey we know the general tremor level of the patient, and from the interview results we know do they have tremor in hands, and which hand they use for playing. When we investigate the game score variation in different sensitivity levels (Fig. 5), we see that the mean score decreased when the sensitivity increased for two patients (P3 and P8). However, the effect of sensitivity change cannot be observed in all participants' scores.

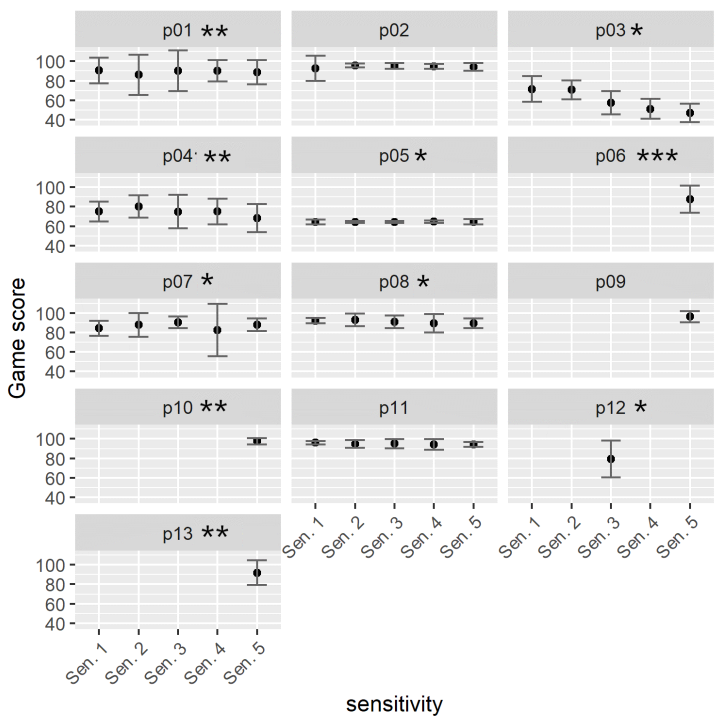

Figure 5: Game component score results in range 0-100 ( $y-$ axis) with different sensitivity levels $1-5$ (x-axis). Stars denote tremor severity according to the UPDRS survey: * - light tremor, ** - mild, *** - moderate.

To investigate how the game score is related with tremor, we computed the Kendall tau-b correlation between game score and sensitivity on a per participant 
basis (Table 1). Significant negative correlations were found for five participants, suggesting that as the sensitivity of the ball increases, people got lower scores. In the interviews, the slowest ball speed (sensitivity 1) was reported to be too easy. Stress, tiredness and agitation were considered to decrease the scores, and concentrating to increase.

"I got usually quite similar scores, but sometimes I noticed I got a little lower score if I had for example stress." (P2)

Nunes and Fizpatrick [14] similarly discovered that emotions can affect the symptom level in PD. Negative feelings, such as being nervous or upset, made the symptoms stronger, and feeling calm improved ability to perform from different activities. Game sessions were experienced effortless, a participant told that the game session does not disrupt much, integrating into his daily routines. On the other hand, the participants were required to play the game at least four times per day during the study, and the scores for all patients were quite high. Perhaps the game was too easy. Our plan is to make the sensitivity and game length configurable. We got feedback describing the game started to feel a bit boring in the end. P6 used iPad for playing the game, and the phone for medication logging.

"The game is expected to be played several times per day, it is hard to keep the device with you always. The application was boring. If I had to use this longer, there could be more games." (P6)

Three participants told in the interviews that it was difficult to reply to the daily survey. Sometimes you don't know what disease is causing the symptoms.

"Daily query is sometimes hard to answer, since I have osteoporosis and tumor in parathyroid (maybe caused by PD medication). You cannot always tell which disease causes the symptoms." (P1)

Our initial hypothesis was that STOP would help PD patients to better manage their medication intake. In the final interview, many of our participants did not feel that they benefited using STOP for that purpose. All participants had been diagnosed with PD at least a few years, hence they had a routine for taking care of medication - some used alarm clocks, some had otherwise firm daily routines, thus they did not feel a need for an application. One of the participants (P1) said that main benefit of using the application was joy of success.

"Yes, there was a benefit, it was nice to be part of a study, and I was able to follow my performance. I thought that even though I have tremor and have continuous medication, I was able to succeed in this, and the good scores encouraged me. I didn't feel failure when I got lower score sometimes." (P1)

\section{Conclusions}

We have significant insight into future work for STOP. Our participants were motivated to measure and quantify their PD condition and symptoms, reporting "a feeling of control." All participants would be comfortable with showing the data collected by the application for a doctor if it helped in the treatment. The possibility to share the data was additional motivation to use STOP to measure their daily symptoms by playing a game and consistently tracking the medication.

\section{Acknowledgements}

This work is partially funded by the Academy of Finland (Grants 313224-STOP, 316253-SENSATE). 


\section{References}

1. mPower 2.0. Description of the study. Accessed from https://parkinsonmpower.org/about

2. mPower Public Reseacher Portal. Accessed from https://www.synapse.org/\#!Synapse:syn4993293/ wiki/394516

3. Sergi Barrantes, Antonio J Sánchez Egea, Hernán A González Rojas, Maria J Martí, Yaroslau Compta,

Francesc Valldeoriola, Ester Simo Mezquita, Eduard Tolosa and Josep Valls-Solè. 2017. Differentia diagnosis between Parkinson's disease and essential tremor using the smartphone's accelerometer. PloS one, 12 (8). e0183843.

4. Brian M Bot, Christine Suver, Elias Chaibub Neto, Michael Kellen, Arno Klein, Christopher Bare, Megan Doerr, Abhishek Pratap, John Wilbanks and E Ray Dorsey. 2016. The mPower study, Parkinson disease mobile data collected using ResearchKit. Scientific data, 3. 160011.

5. Parkinson Association of the Carolinas, Statistics on Parkinson's Disease. Accessed from https://www.parkinsonassociation.org/facts-aboutparkinsons-disease/

6. S.R.L.E. Fahn. 1987. Unified Parkinson's disease rating scale. Recent development in Parkinson's disease.

7. Donald Grosset, Angelo Antonini, Margherita Canesi, Gianni Pezzoli, Andrew Lees, Karen Shaw Esther Cubo, Pablo Martinez-Martin, Olivier Rascol and Laurence Negre-Pages. 2009. Adherence to antiparkinson medication in a multicenter European study. Movement disorders: official journal of the Movement Disorder Society, 24 (6). 826-832.

8. Valerii Kan. 2018. STOP: A smartphone-based game for Parkinson's disease medication adherence Faculty of Information Technology and Electrical Engineering, University of Oulu, Oulu, 52.
9. Valerii Kan, Dorina Rajanen, Kennedy Opoku Asare, and Denzil Ferreira. 2018. STOP: A Smartphonebased Game for Parkinson's Disease Medication Adherence. In Proceedings of the 2018 ACM International Joint Conference and 2018 International Symposium on Pervasive and Ubiquitous Computing and Wearable Computers (UbiComp '18). ACM, New York, NY, USA, 373-376. DOI: https://doi.org/10.1145/3267305.3267598

10. Rashmi Lakshminarayana, Duolao Wang, David Burn, K. Ray Chaudhuri, Clare Galtrey, Natalie Valle Guzman, Bruce Hellman, James Ben, Suvankar Pal, Jon Stamford, Malcolm Steiger, R. W. Stott, James Teo, Roger A. Barker, Emma Wang, Bastiaan R. Bloem, Martijn van der Eijk, Lynn Rochester and Adrian Williams. 2017. Using a smartphone-based self-management platform to support medication adherence and clinical consultation in Parkinson's disease. npj Parkinson's Disease, 3 (1). 2. DOI: https://doi.org/10.1038/s41531-016-0003-z

11. Robert LeMoyne, Timothy Mastroianni, Michael Cozza, Cristian Coroian and Warren Grundfest. Year. Implementation of an iPhone for characterizing Parkinson's disease tremor through a wireless accelerometer application. In Engineering in Medicine and Biology Society (EMBC), 2010 Annual International Conference of the IEEE, IEEE, 4954-4958.

12. Edward Loper and Steven Bird. Year. NLTK: The natural language toolkit. In Proceedings of the ACL02 Workshop on Effective tools and methodologies for teaching natural language processing and computational linguistics-Volume 1, Association for Computational Linguistics, 63-70.

13. Elias Chaibub Neto, Thanneer M Perumal, Abhishek Pratap, Brian M Bot, Lara Mangravite and Larsson Omberg. 2017. On the analysis of personalized medication response and classification of case vs control patients in mobile health studies: the 
mPower case study. arXiv preprint arXiv: 1706.09574.

14. Francisco Nunes and Geraldine Fitzpatrick. Year. Understanding the Mundane Nature of Self-care:

Ethnographic Accounts of People Living with

Parkinson's. In Proceedings of the $2018 \mathrm{CHI}$ Conference on Human Factors in Computing

Systems, ACM, 402.

15. The Michael J. Fox Foundation for Parkinson's Research, Parkinson's Disease

Medications. Accessed from

https://www.michaeljfox.org/understandingparkinsons/living-with-

pd/topic. php?medication\&navid=medication

16. Alan Michael Woods, Mariusz Nowostawski, Elizabeth A Franz and Martin Purvis. 2014 Parkinson's disease and essential tremor classification on mobile device. Pervasive and Mobile Computing, 13. 1-12. 